\title{
Christian Fontaine, Philippe Liverneaux, Emmanuel Masmejean and Jean-Yves Alnot (eds.): Cours européen de pathologie chirurgicale du membre supérieur et de la main-2009
}

\author{
Sauramps Médical 2009, 547 pp, Euro 76.00, ISBN: 978-2840236047
}

\section{Pierre Kehr}

Received: 28 February 2010 / Accepted: 1 March 2010 / Published online: 13 March 2010

(c) Springer-Verlag 2010

Il s'agit d'un livre multi auteurs reprenant le cours annuel.

La première partie concerne les raideurs post traumatiques de la main mais aussi du coude, de l'épaule mais également des synostoses radio ulnaires. Cette partie permet de faire le point sur des lésions pas toujours faciles à traiter et dont le résultat n'est pas toujours aussi bon que l'on pourrait l'espérer.

La deuxième partie concerne les syndromes canalaires, des plus fréquents comme le canal carpien aux pathologies plus rares comme la pathologie du nerf thoracique long ou le syndrome de Parsonage et Turner. Cette partie permet de faire le point et de découvrir ou de redécouvrir des lésions rares.
La troisième partie concerne la traumatologie ostéoarticulaire de l'enfant faisant ressortir les particularités des traumatismes à ce niveau.

Un livre intéressant qui concernera autant les jeunes chirurgiens, qui trouveront le rappel des notions de base, que les chirurgiens plus expérimentés qui découvriront des techniques pointues.

Alain Graftiaux Strasbourg (France)

No funds were received in support of this study. 